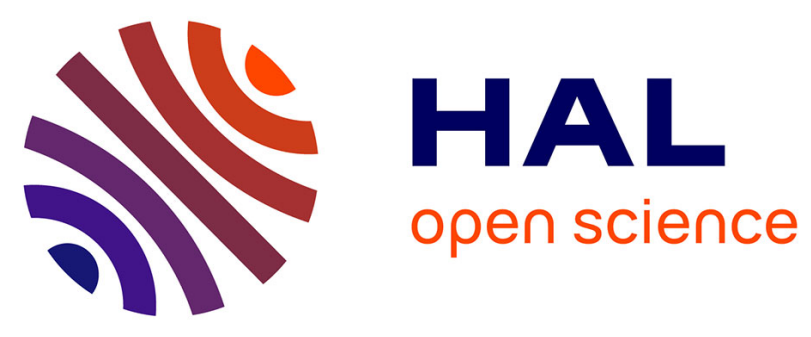

\title{
Should I stay or should I go now?Recovery time effect on walking capacity in symptomatic peripheral artery disease
}

\author{
Pierre-Yves de Müllenheim, Laurent Rouvière, Mathieu Emily, Ségolène \\ Chaudru, Adrien Kaladji, Guillaume Mahé, Alexis Le Faucheur
}

\section{To cite this version:}

Pierre-Yves de Müllenheim, Laurent Rouvière, Mathieu Emily, Ségolène Chaudru, Adrien Kaladji, et al.. Should I stay or should I go now?Recovery time effect on walking capacity in symptomatic peripheral artery disease. Journal of Applied Physiology Respiratory Environmental and Exercise Physiology, 2021, 131 (1), pp.207-219. 10.1152/japplphysiol.00441.2020 . hal-03246582

\section{HAL Id: hal-03246582 https://hal.science/hal-03246582}

Submitted on 15 Jun 2021

HAL is a multi-disciplinary open access archive for the deposit and dissemination of scientific research documents, whether they are published or not. The documents may come from teaching and research institutions in France or abroad, or from public or private research centers.
L'archive ouverte pluridisciplinaire HAL, est destinée au dépôt et à la diffusion de documents scientifiques de niveau recherche, publiés ou non, émanant des établissements d'enseignement et de recherche français ou étrangers, des laboratoires publics ou privés. 
4 Short title: Recovery time and walking capacity in PAD

6 Author list: Pierre-Yves de Müllenheim ${ }^{1}$; Laurent Rouvière ${ }^{2}$; Mathieu Emily ${ }^{3}$; Ségolène

7 Chaudru $^{4,5}$; Adrien Kaladji ${ }^{6}$; Guillaume Mahé ${ }^{4,5,7}$; Alexis Le Faucheur ${ }^{4,8}$

\section{Affiliations}

$10{ }^{1}$ Institute of Physical Education and Sports Sciences (IFEPSA), West Catholic University

11 (UCO), F-49136, Les Ponts-de-Cé, France.

$12{ }^{2}$ Univ Rennes, IRMAR - UMR CNRS 6625, F-35000, Rennes, France.

$13{ }^{3}$ Institut Agro, CNRS, Univ Rennes, IRMAR -UMR 6625, F-35000 Rennes, France.

$14{ }^{4}$ Clinical Investigation Center, INSERM CIC 1414, F-35033, Rennes, France.

$15{ }^{5}$ Univ Rennes, F-35000, Rennes, France.

$16{ }^{6}$ Department of Cardiothoracic and Vascular Surgery, University Hospital Rennes, F-35000,

17 Rennes, France.

$18{ }^{7}$ Vascular medicine unit, University Hospital Rennes, F-35000, Rennes, France.

$19{ }^{8}$ Univ Rennes, M2S - EA 7470, F-35000, Rennes, France.

21 Acknowledgements

22 This work was promoted and partially funded by the University Hospital of Rennes

23 (CORECT 2013). This work was also partially funded by ENS Rennes. S. Chaudru received 24 a PhD scholarship from the Bretagne region (Bourse ARED). P.-Y. de Müllenheim received 25 a PhD scholarship from the ENS Cachan-Antenne de Bretagne (CDSN). 
26 A.L.F., G.M., and P.-Y.d.M. conceived and designed the study; P.-Y.d.M., SC, A.L.F., and

27 G.M. performed the experiments; L.R., M.E., P.-Y.d.M., and A.L.F. analyzed the data; P.-

28 Y.d.M. and A.L.F. interpreted the results of the experiments; P.-Y.d.M. prepared the figures;

29 P.-Y.d.M. and A.L.F. drafted the manuscript; P.-Y.d.M., L.R., M.E., S.C., G.M., A.K. and

30 A.L.F. edited and revised the manuscript; P.-Y.d.M., L.R., M.E., S.C., G.M., A.K. and A.L.F.

31 approved the final version of the manuscript.

\section{Conflict of interest}

34 The authors declare no conflict of interest.

35

36 Corresponding author

37 Alexis LE FAUCHEUR. Department of Sport sciences and physical education, ENS Rennes,

38 Campus de Ker Lann, Bruz, F-35170, France. Tel: +33 (0)2 9905 9419. Fax: +33 (0)2 9905

39 9329. E-mail: alexis.lefaucheur@ens-rennes.fr

40 


\section{ABSTRACT}

42 Objective: To investigate the effect of recovery time on walking capacity (WC) throughout

43 repeated maximal walking bouts in symptomatic lower extremity peripheral artery disease 44 (PAD).

45 Methods: The effect of recovery time on WC (maximal walking time) was determined in 21

46 participants with PAD in three experimental conditions (recovery time from $0.5-9.5 \mathrm{~min}+\mathrm{a}$

47 self-selected recovery time [SSRT]): (i) 11 repeated sequences of two treadmill walking

48 bouts (TW-ISO); (ii) a single sequence of seven treadmill walking bouts (TW-CONS); (iii) a

49 single sequence of seven outdoor walking bouts (OW-CONS). Exercise transcutaneous

50 oxygen pressure changes were continuously recorded as an indirect measure of ischemia. An

51 individual recovery time (IRT) beyond which WC did not substantially increase was

52 determined in participants with a logarithmic fit.

53 Results: At the group level, mixed models showed a significant effect $(P<0.001)$ of recovery

54 time on WC restoration. At the participant level, strong logarithmic relationships were found 55 (median significant $\mathrm{R}^{2} \geq 0.78$ ). The median SSRT corresponded to a median work-to-rest ratio $56>1: 1$ (i.e., a lower recovery time in view of the corresponding previous walking time) and 57 was related to unrecovered ischemia and a WC restoration level of $<80 \%$. A median work-to58 rest ratio of $\leq 1: 2$ allowed full recovery of ischemia and full restoration of WC. The IRT ratio

59 was between 1:1 and 1:2 and corresponded to the start of recovery from ischemia.

60 Conclusion: Recovery time affects the restoration level of WC during repeated maximal

61 walking bouts in symptomatic PAD. Meaningful variations in WC restoration were related to 62 specific levels of work-to-rest ratios.

63

64 Key Words: intermittent claudication, ischemia, walking, exercise, work-to-rest ratio, 65 exercise therapy. 


\section{NEW \& NOTEWORTHY}

67 This study demonstrated there is a significant and mostly logarithmic effect of recovery time 68 on walking capacity in people with symptomatic PAD. This study revealed that a median

69 work-to-rest ratio $>1: 1$ leads to the resumption of walking with unrecovered ischemia and

70 precludes the restoration of full walking capacity, whereas a work-to-rest ratio $\leq 1: 2$ allowed 71 walking capacity to fully be restored. 


\section{INTRODUCTION}

73 Lower extremity peripheral artery disease (PAD) accounts for a considerable public health

74 burden, with more than 236 million people aged $\geq 25$ years living with PAD worldwide (49).

75 Lower-limb pain during walking, caused by ischemia in active muscles (50), leads to an

76 impaired walking capacity (15), which decreases quality of life compared with that in people

77 without PAD (46) and independently predicts mortality (32). Supervised, or, when not

78 available, home-based or community-based walking programs are recommended as the first-

79 line therapy to improve functional status and quality of life and to reduce leg symptoms in 80 people with PAD (22).

82 The optimal exercise regimen for walking programs for people with PAD has been published

83 in several authoritative scientific reports $(3,4,52)$. However, the current recommendations

84 fail to provide clear indications regarding the optimal work-to-rest ratio that should be 85 prescribed $(6,22,52)$. This missing information is directly related to the fact that the 86 recovery pattern and the variation in walking performance throughout repeated walking bouts

87 limited by pain-related ischemia, either in treadmill or outdoor walking conditions, have 88 never been studied. Interestingly, from global positioning system (GPS) measurements, we 89 previously observed that the stop duration between two pain-limited walking bouts could be 90 inversely associated with the walking capacity of people with PAD during an outdoor 91 walking session including multiple walking bouts (31). While this previous study provided 92 some insights into the potential effect that recovery time (RT) may have on walking capacity, 93 to our knowledge, no studies have experimentally or systematically determined whether and 94 how RT predicts the walking capacity of people with PAD during repeated treadmill or 95 outdoor walking bouts. 
97 Thus, the first aim of the present study was to determine the effect of RT on walking capacity

98 in people with symptomatic PAD throughout repeated walking bouts performed up to 99 maximal claudication pain, during both treadmill and outdoor walking conditions. Such an

100 approach is a preliminary and required step before implementing and testing future walking

101 training protocols that could be optimized based on different work-to-rest ratio strategies. We

102 hypothesized here that RT would predict the walking capacity through either a logarithmic or

103 a linear relationship (31). Furthermore, from these relationships, together with the study of

104 the time course of ischemia during recovery, a second aim was to explore the possibility of 105 identifying specific levels of work-to-rest ratios that would be associated with meaningful 106 variations in terms of walking capacity restoration. 


\section{Study design}

109 The CLASH protocol is a cross-sectional study that was approved by our local ethics

110 committee (CPP OUEST V, Rennes, France) and registered with the American National

111 Institutes of Health database under reference No. NCT02041169.

112 Participants were initially referred by their physician or angiologist to our Vascular Medicine

113 Unit (University Hospital, Rennes, France) for a treadmill evaluation of walking capacity.

114 After screening, they were invited to participate in the study if they fulfilled the inclusion

115 criteria (Table 1). All included participants provided their written informed consent to

116 participate. Then, within a $\sim 1$-month period, the included participants with PAD first

117 underwent an inclusion visit followed by a 7-day free-living measurement of physical activity

118 using wearable monitors (not reported here; see Chaudru et al. (7)). Thereafter, the

119 participants were underwent testing in the three distinct experimental conditions described in

120 Figure 1.

121

\section{Inclusion visit}

123 Physical characteristics, cardiovascular risk factors, comorbid conditions, claudication history

124 and the list of current medications were obtained through the medical history and medical

125 reports. Participants were asked to estimate the distance they could walk at the usual pace on

126 a flat surface until lower-limb pain forced them to stop. Self-reported ambulatory walking

127 impairment was also assessed using the walking impairment questionnaire (WIQ) (37).

128 Quality of life was evaluated using the SF-36 questionnaire (33). Lower-limb symptoms were

129 assessed and classified using the San Diego claudication questionnaire (9). The resting ankle

130 brachial index (ABI) was measured using a handheld Doppler probe according to the

131 recommendations of the American Heart Association (1). Then, participants with PAD 
132 performed a Gardner-Skinner treadmill test $(3.2 \mathrm{~km} / \mathrm{h}, 0 \%$ grade with a $2 \%$ increase every 2 133 min) until maximal claudication pain.

\section{Experimental conditions}

136 Procedures. The effect of RT on walking capacity was investigated using three distinct

137 experimental conditions (Figure 1): (i) isolated sequences of treadmill maximal walking 138 bouts (TW-ISO); (ii) consecutive treadmill maximal walking bouts (TW-CONS); and (iii) 139 consecutive outdoor maximal walking bouts (OW-CONS). Furthermore, to assess the 140 outdoor walking capacity of the participants with PAD without controlling the effect of RT,

141 participants performed another outdoor walking session on another day, as previously 142 reported $(29,31)$.

143 For the three experimental conditions, the aim was to determine the effect of RT on walking 144 capacity by manipulating the RT following an ischemia-limited walking bout and by 145 measuring the walking time of the subsequent walking bout performed up to maximal 146 claudication pain. The fixed RTs tested were $<10 \mathrm{~min}$, which was consistent with the 147 definition of intermittent claudication (8) and previous observation during outdoor walking 148 (23). In addition to fixed RTs, each experimental condition included a self-selected RT 149 (SSRT) where each participant was asked to resume the walk once he/she felt it was possible, 150 as he/she would have done in daily life. Furthermore, for all walking bouts in the three 151 experimental conditions, we required participants to walk to maximal claudication to make 152 the level of ischemia reached more comparable (at the maximal endpoint). The specific 153 rationale and protocol for each condition are as follows:

154 (i) TW-ISO. To isolate, as much as possible, the potential effect of RT on treadmill 155 walking capacity, participants performed 11 separated sequences of treadmill walking 156 (Figure 1). A sequence included two walking bouts $(3.2 \mathrm{~km} / \mathrm{h}, 12 \%$ grade) until 
maximal claudication pain, interspersed by a given RT (between 0.5 and $9.5 \mathrm{~min}$; or SSRT). No more than three sequences were performed during a session, and they were separated by $\geq 15$ min of recovery to retrieve baseline values of oxygen uptake $\left(\dot{\mathrm{VO}}_{2}\right)$ and transcutaneous oxygen pressure changes $\left(\mathrm{TcPO}_{2}\right)$. The 11 sequences were randomly spread over four sessions on four different days (3 sessions with 3 162 sequences and 1 session with 2 sequences).

(ii) TW-CONS. The rationale here was to assess the potential effect of RT on walking capacity through a series of consecutive walking bouts, thereby moving toward what was previously observed during outdoor walking in PAD (31). Thus, during one single session, participants performed seven consecutive treadmill walking bouts (3.2 $\mathrm{km} / \mathrm{h}, 12 \%$ grade) up to maximal claudication pain, interspersed by six RTs (between 0.5 and $8.5 \mathrm{~min}$; or SSRT).

(iii) OW-CONS. The rationale here was close to that explained for TW-CONS except that here, we aimed to reproduce the walking pattern of people with PAD under "natural" outdoor walking conditions, as encountered while walking during daily life (31) or during outdoor walking sessions of a home-based walking program (16). Thus, during a single session, participants performed seven consecutive outdoor (athletic track; $0 \%$ grade) walking bouts up to maximal claudication pain, interspersed by six RTs (between 0.5 and $4.5 \mathrm{~min}$; or SSRT). Consistent with previous observation (23), RTs were intentionally shorter than those for TW-ISO and TW-CONS. Participants were asked to walk at their usual walking speed and to stop walking only due to maximal claudication pain. The outdoor walking session was supervised by two investigators who walked approximately 10 meters behind the participant and controlled the RTs. 
181 Randomization. The order of the experimental conditions was randomized, with the two

182 outdoor walking sessions performed consecutively. All walking sessions were separated by at

183 least 48 hours. Within each experimental condition, the RTs were also randomized, and 184 participants were blinded to the RTs tested.

186 Instrumentation \& measurement

187 Treadmill walking. Participants were blinded to the distance and time walked. Participants

188 were permitted to touch the handrail of the treadmill using their hands or fingers only to

189 maintain balance. Moreover, they were asked to orally indicate to the investigator the

190 claudication pain score they reached during both walking and recovery based on a 5-point

191 scale $(0=$ no pain; $1=$ onset of pain; $2=$ moderate pain; $3=$ intense pain; $4=$ maximal pain $)$

192 (3). The treadmill walking bouts were stopped when maximal claudication pain was reached.

193 During each recovery bout, the participants were asked about the localization of the lower-

194 limb pain.

195 Cardiorespiratory variables. Gas exchange and heart rate (HR) were measured during the

196 Gardner-Skinner treadmill test and all the experimental conditions. A metabolic cart

197 (MasterScreen CPX [MSCPX], Jaeger®, Germany) was used during treadmill walking, and a

198 portable metabolic system (K4b², Cosmed ${ }^{2}$, Italy) was used during outdoor walking. For

199 measuring HR, the MSCPX used a 12-lead electrocardiogram, while the $\mathrm{K}_{4} \mathrm{~b}^{2}$ used a Polar ${ }^{\circledR}$

200 belt. The systems were calibrated according to the manufacturer's instructions before each

201 walking session.

202 Exercise transcutaneous oxygen pressure $\left(\right.$ Exercise- $\left.\mathrm{TcPO}_{2}\right)$. Exercise- $\mathrm{TcPO}_{2}$ was

203 continuously measured during the Gardner-Skinner treadmill test and the treadmill sessions.

204 Measurements were performed using five probes positioned on the chest, the two buttocks, 
and the two calves, as previously described $(35,44)$. Exercise- $\mathrm{TcPO}_{2}$ was used as an indirect measure of exercise-induced ischemia $(2,51)$.

207 Outdoor walking. For the two outdoor walking sessions, the participants were also equipped

208 with a set of physical activity monitors (not reported here; see Chaudru et al. (7)), including a

209 DG100 GPS receiver (GlobalSat ${ }^{\circledR}$, Taiwan) (41), which was used to obtain walking times

210 and distances, and a digital watch, which allowed the participant to indicate when pain

211 occurred during walking (7). The walking times and distances were obtained from the

212 walking bouts that were detected using the GPS speed signals along with a validated data

213 processing methodology $(30,41)$. During the walking session, using a chronometer, the

214 investigators reported the time when the participant pressed the marker button on the watch

215 to indicate the occurrence of pain (the watch data were not used in the present study, see

216 Chaudru et al. (7)).

\section{Data analysis}

219 Questionnaire outcomes. The responses to the WIQ and SF-36 questionnaires were recorded

220 in a spreadsheet and were scored following the recommended procedures $(9,33,37)$.

221 Walking capacity outcomes. For each treadmill walking bout, we computed the pain-free 222 walking times (PFWTs) and the maximal walking times (MWTs). From each outdoor 223 walking bout, the PFWT was the duration measured by the investigators, using the 224 chronometer, from the start of the walking bout to the moment where the participant 225 indicated the occurrence of pain using the watch provided for this purpose (7). The MWT 226 was the duration of the maximal walking bout detected using the GPS as previously reported $227(7,29)$.

228 Physiological outcomes. Oxygen uptake $\left(\mathrm{V}_{2}\right)$ and $\mathrm{HR}$ data were time-averaged to 5-s 229 intervals. Data obtained during the experimental conditions are expressed as percentages of 
230 peak $\dot{\mathrm{VO}}_{2}\left(\% \dot{\mathrm{V}} \mathrm{O}_{2 \text { peak }}\right)$ and $\mathrm{HR}\left(\% \mathrm{HR}_{\text {peak }}\right)$. Since peak physiological variables can exhibit 231 some variability, $\dot{\mathrm{V}} \mathrm{O}_{2 \text { peak }}$ and $\mathrm{HR}_{\text {peak }}$ were the highest 15 -s average values of $\dot{\mathrm{V}} \mathrm{O}_{2}$ and $\mathrm{HR}$ 232 measured throughout the entire study protocol, respectively. We used an unpublished 233 treadmill equation to make the $\dot{\mathrm{VO}}_{2}$ data measured with the $\mathrm{K}_{4} \mathrm{~b}^{2}$ comparable with the $234 \mathrm{MSCPX} \dot{\mathrm{VO}}_{2}$ data. Indeed, our unpublished data showed that the $\mathrm{K}_{4} \mathrm{~b}^{2}$ overestimated $\dot{\mathrm{VO}}_{2}$ 235 compared with the MSCPX. The protocol and associated results, including the correction 236 equation, are detailed in Supplemental Digital Content 1.

237 For the Exercise- $\mathrm{TcPO}_{2}$ measurement, the delta values from the resting oxygen pressure 238 (DROP) values (in $\mathrm{mmHg}$ ) at each site were computed every $2.5 \mathrm{~s}$ as the changes in $\mathrm{TcPO}_{2}$

239 from the 2-min resting mean value preceding the series of walking bouts minus the 240 corresponding absolute change in $\mathrm{TcPO}_{2}$ recorded at the chest $(35,44)$.

241 For all experimental conditions, end-walking and end-recovery physiological values were 242 computed by averaging the last three-time intervals of the period. All these calculations were 243 performed using Excel $\mathbb{R}($ Microsoft $\AA$, Richmond, VA) spreadsheets.

\section{Statistical analysis}

246 All statistical analyses were performed using R software (45). The normality of continuous 247 variables was assessed using the Shapiro-Wilk test. The mean and standard deviation (SD)

248 were used when the data followed a normal distribution; otherwise, medians and $25^{\text {th }}-75^{\text {th }}$ 249 percentiles were used. The effects and differences tested during the statistical analysis were 250 considered significant when $P \leq 0.05$.

251 Effect of RT on walking capacity. To test the hypothesis that RT would predict walking 252 capacity, for each experimental condition, we first built linear mixed models at the group 253 level, followed by models for each participant. In both cases, the response variable was the 254 ratio of MWT performed after a given RT $\left(\mathrm{MWT}_{\mathrm{n}}\right)$ divided by the first MWT of the sequence 
$255\left(\mathrm{MWT}_{1}\right)$, and the explanatory variable was either (i) the RT (x) or (ii) the logarithm of RT

$256(\ln (\mathrm{x}))$. The $\mathrm{MWT}_{\mathrm{n}} / \mathrm{MWT}_{1}$ ratio was considered as the restoration level of the MWT of the

257 participants. The use of a logarithm model was motivated by previous observation of the

258 association of RT and walking capacity during outdoor walking sessions (31). At both the

259 group and participant (individual) levels, data related to SSRT were not included in the

260 models. In addition, for both the TW-CONS and OW-CONS conditions, we calculated the

261 cumulated time of walking (from the beginning of the session) as an indirect estimate of

262 fatigue (31). Then, at the group level, from the best model (using RT (x) or RT $(\ln (\mathrm{x})$ )

263 obtained, we performed a step-by-step multivariate linear regression analysis with stepwise

264 selection to determine whether the addition of the cumulated time could also be a predictor of

265 the walking capacity. For OW-CONS, we also tested the potential effect of adding the

266 walking speed in the multivariate analysis (31). Furthermore, at the individual level, the

267 potential associations between walking capacity and each of these potential predictors were

268 also investigated separately using correlation analyses. For this purpose, to take into account

269 possible multicollinearity effects, significant associations between the cumulated time or

270 walking speed (independent variables) and walking capacity were not considered if a

271 correlation also existed between the independent variable tested and RT. $\mathrm{R}^{2}$ coefficients for

272 all models, root-mean-squared error (RMSE) for the mixed models, and standard error of

273 estimate (SEE) for individual models were calculated to assess the goodness of fit and

274 accuracy of the models.

275 Determination of an individual RT (IRT). For each experimental condition in participants

276 who exhibited a plausible logarithmic effect of RT on $\mathrm{MWT}_{\mathrm{n}} / \mathrm{MWT}_{1}$ (i.e., with $\mathrm{P} \leq 0.05$ for

277 the slope and $\mathrm{R}_{\ln (\mathrm{x})}^{2}>\mathrm{R}_{\mathrm{x}}^{2}$ ), we investigated the presence of a possible IRT that could enable

278 these participants to "maximize" their walking capacity after a symptom-limited walking

279 bout. Conceptually, below the IRT, a small increase in RT should substantially increase the 
280 MWT. Conversely, with an RT greater than the IRT, the MWT should increase much more

281 slowly despite an important increase in RT. The IRT would be close to the breakpoint 282 between the two lines of distinct slopes that fit the logarithmic curve related to the participant 283 data. Thus, to determine the IRT for each experimental condition, we used the segmented 284 linear regression model developed by Muggeo (39). This model assumes that the relationship 285 between the response variable (i.e., $\mathrm{MWT}_{\mathrm{n}} / \mathrm{MWT}_{1}$ ) and the explanatory variable (i.e., RT) is 286 piecewise linear. In other words, we considered two linear models separated by a breakpoint, 287 and the IRT corresponded to the estimator of this breakpoint. An example illustrating the 288 procedure implemented to determine the IRT in one participant is shown in Figure 2.

289 Among the participants who exhibited a plausible linear effect of RT on $\mathrm{MWT}_{\mathrm{n}} / \mathrm{MWT}_{1}$ (i.e., 290 with $P \leq 0.05$ for the slope and $\mathrm{R}_{\mathrm{x}}^{2}>\mathrm{R}_{\ln (\mathrm{x})}^{2}$ ), IRT was assigned to the participants with the 291 highest RT tested in the considered experimental condition, assuming a possible 292 underestimation of the IRT. The participants who did not exhibit a significant effect of RT on $293 \mathrm{MWT}_{\mathrm{n}} / \mathrm{MWT}_{1}$ were not considered for the determination of IRT.

294 For each treadmill condition, the time needed to reach a pain level of "0", SSRT, and IRT 295 were compared using a Friedman test. If significant, pairwise comparisons were performed 296 using two-sided Wilcoxon signed rank tests with $P$ value adjustment (Bonferroni correction).

297 For the TW-ISO and TW-CONS conditions, the values used for the time needed to reach a 298 pain level of " 0 " were the participant averages of the times measured during the corresponding conditions (this time was not measured during the OW-CONS condition). In

300 the OW-CONS condition, SSRT and IRT were compared using a two-sided Wilcoxon signed 301 rank test.

302 For each RT in each condition and based on participants in whom there was a plausible 303 logarithmic or linear effect of RT on walking capacity, we calculated the corresponding 
304 median work-to-rest ratio. This ratio was first calculated by dividing the considered RT by

305 the previous MWT of the participant and then by taking the median of the participant ratios.

306 Comparison of the restoration levels of walking capacity and physiological values between

307 the RTs tested. For each experimental condition, we investigated the RTs for which the

$308 \mathrm{PFWT}_{\mathrm{n}} / \mathrm{PFWT}_{1}$ and $\mathrm{MWT}_{\mathrm{n}} / \mathrm{MWT}_{1}$ ratios, as well as the end-recovery $\% \dot{\mathrm{VO}}_{2 \text { peak }}, \% \mathrm{HR}_{\text {peak }}$,

309 and ischemia levels, were likely to no longer change. For this purpose, pairwise comparisons

310 between two successive RTs were performed using a one-sided Wilcoxon signed rank test.

311 All calculations were performed using the participants who exhibited either a linear or

312 logarithmic effect of RT on walking capacity associated with a $P$ value of $\leq 0.05$ for the slope. 


\section{RESULTS}

314 Twenty-three participants with PAD were initially included in the study, but two were

315 excluded before the end of the study. One participant was excluded due to a need for medical

316 treatment modification, and another participant stopped participating to undergo a surgical

317 intervention. Thus, data from 21 participants were analyzed in the present study. The

318 participants' characteristics are shown in Table 2. Participants were mainly older,

319 overweight, Caucasian men with a resting ABI indicating a mild-to-moderate PAD severity

$320(0.67 \pm 0.17)$. All participants were limited by exertional lower-limb symptoms during the

321 Gardner-Skinner test at the inclusion visit.

\section{Effect of RT on walking capacity}

324 A total of 742 walking bouts were performed ( $\mathrm{n}=462$ for TW-ISO; $\mathrm{n}=146$ for TW-CONS;

$325 \mathrm{n}=134$ for OW-CONS). All 21 participants completed the 11 sequences of the TW-ISO

326 condition. For the TW-CONS condition, one participant was unable to complete the last

327 walking bout of the session (that would have followed the SSRT) because of fatigue. For the

328 OW-CONS condition, one participant was not pain-limited (no RT tested), and three other

329 participants did not perform their two last maximal walking bouts because the duration of the

330 walking session would have exceeded the a priori fixed 1-h limit. The mean $( \pm \mathrm{SD})$ of the

331 session mean speed was $4.4 \pm 0.4 \mathrm{~km} / \mathrm{h}$.

332 Linear mixed models testing the effect of RT on the $\mathrm{MWT}_{\mathrm{n}} / \mathrm{MWT}_{1}$ ratio at the group level

333 are shown in Table 3. Regardless of the model tested and the experimental conditions, the RT

334 significantly predicted the $\mathrm{MWT}_{\mathrm{n}} / \mathrm{MWT}_{1}$ ratio $(P<0.001)$. Although the explained variance

335 remained quite low $\left(\mathrm{R}^{2}\right.$ between 0.19 and 0.45 ), a greater $\mathrm{R}^{2}$ was obtained using the

336 logarithm of the RT as the explanatory variable. Furthermore, neither the cumulated time

337 (TW-CONS \& OW-CONS) nor the walking speed (OW-CONS) was found to be a predictor 
338 of walking capacity $(P>0.05)$. At the individual level (Table 4$)$, the effect of RT on walking

339 capacity $\left(\mathrm{MWT}_{\mathrm{n}} / \mathrm{MWT}_{1}\right)$ was significant in most participants $(\geq 70 \%)$ regardless of the

340 condition tested, and this effect was logarithmic rather than linear in most cases $(n=16 / 21$,

341 16/21 and 11/21 for TW-ISO, TW-CONS and OW-CONS, respectively). For participants

342 who exhibited a logarithmic effect, the median $\mathrm{R}^{2}$ was high $(0.80)$ in the TW-ISO condition

343 to very high $(>0.90)$ in the TW-CONS and OW-CONS conditions. For TW-CONS, a

344 significant negative association $(P=0.011)$ between walking capacity and cumulated time was

345 found in only one participant who otherwise did not show an effect of RT.

346 The effect of RT on walking capacity in each of the three experimental conditions is

347 illustrated in Figure 2. As shown, during TW-ISO, the MWT tended to be fully (100\%)

348 restored from $4.5 \mathrm{~min}$ of recovery (91.4\% [86.7- 105.4]). During TW-CONS, the MWT

349 tended to be fully restored from $6.5 \min (88.2 \%[75.6-96.9])$. We observed that during the

350 outdoor walking condition, most of the participants $(9 / 14)$ were unable to restore $\geq 80 \%$ of

351 their MWT after 4.5 min of recovery, whereas this was less often the case during treadmill

352 walking conditions (3/18 for TW-ISO, 7/17 for TW-CONS). When observing the PFWT, the

353 relationship between the percentage of the restored PFWT and the RT presented a sigmoidal

354 shape, as several participants still had pain when they resumed walking after short RTs,

355 particularly in the OW-CONS condition.

356 Figure 2 also shows how the physiological parameters were recovered at the end of each of

357 the RTs tested. The main observation was that the percentages of peak $\dot{\mathrm{VO}}_{2}$ and peak HR

358 exponentially decreased as the RT increased. Interestingly, the recovery of DROP values

359 followed a sigmoidal shape as a function of RT. Values were the lowest and the response was

360 blunted until 2.5 min of recovery, whereas the $\mathrm{MWT}_{\mathrm{n}} / \mathrm{MWT}_{1}$ ratio increased over the same

361 period in both treadmill conditions $(P \leq 0.001)$. Thereafter, DROP values increased with RT

$362 \geq 3.5 \mathrm{~min}(P<0.05)$ and finally reached a plateau from $5.5 \mathrm{~min}$ in TW-ISO $(-0.2 \mathrm{mmHg}[-7.9-$ 
363 1.4]) and from $6.5 \mathrm{~min}$ in TW-CONS $(-2.3 \mathrm{mmHg}[-8.7-0.8]$, with a low increase up to 8.5

$364 \mathrm{~min}$ ), indicating full recovery in terms of ischemia. This time needed for full recovery of

365 ischemia was close to that needed for restoring 100\% of the MWT (Figure 3).

366

367 SSRT and IRT

368 For treadmill conditions, the SSRT was not significantly different from the time needed to

369 reach a pain level of " 0 " $(P=1.000)$, and both were lower than IRT $(P<0.001)$, as illustrated in

370 Figure 2. In contrast, for the outdoor condition, the SSRT and IRT were not significantly

371 different $(P=0.391)$.

372 Figure 3 also displays how SSRT and IRT matched the restoration of both MWT and 373 ischemia (DROP values) during the treadmill conditions. As shown, the median SSRT 374 matched the time period during which DROP values were the lowest and the response was 375 blunted (i.e., maximal ischemia), allowing only a restoration of MWT below $80 \%$ in both the 376 TW-ISO and TW-CONS conditions. The median IRT matched the time from which DROP 377 values started to increase (i.e., ischemia decrease), allowing a restoration of MWT to $>90 \%$ 378 in TW-ISO and between $78 \%$ and $86 \%$ in TW-CONS. Finally, when expressing the RTs 379 tested in terms of work-to-rest ratios, it was observed that a median work-to-rest ratio $\geq 1: 1$ 380 did not enable the participants with PAD to start to recover from ischemia (Figure 3), while 381 MWT started to be restored. As shown in Figure 3, the median SSRT was above this 382 threshold of a work-to-rest ratio of 1:1. Conversely, a median work-to-rest ratio of $\leq 1: 2$ 383 seemed to be necessary to enable the participants to fully recover from ischemia and restore $384 \sim 100 \%$ of their MWT. In terms of the work-to-rest ratio, the median IRT was between 1:1 385 and 1:2 and corresponded to an ischemia that started to decrease and to a restoration of MWT 386 to nearly $90 \%$ in TW-ISO and nearly $80 \%$ in TW-CONS. 


\section{DISCUSSION}

388 To our knowledge, the present study is the first to experimentally investigate and demonstrate 389 the effect of RT on walking capacity in people with PAD and to describe the association of

390 RT with ischemic changes in the symptomatic lower limb. There were two main findings.

391 First, by experimentally manipulating the RT, we showed that RT significantly predicts the 392 walking capacity in people with PAD, highlighting two different profiles over the range of 393 the RTs tested (<10 min): a logarithmic profile (for most participants) and a linear profile.

394 The second main finding was the identification of specific levels of work-to-rest ratios that 395 were associated with different levels of MWT restoration.

398 Most of the participants with PAD exhibited a logarithmic effect of RT on walking capacity 399 and were unable to recover $100 \%$ of their walking capacity until ischemia was fully resolved.

400 This finding is consistent with the fact that ischemia impairs force development and thus muscle performance via factor(s) related to blood flow and that are dependent or independent of $\mathrm{O}_{2}$ delivery $(27,43)$. Furthermore, we found that the ischemia level at the end of the recovery was linked to the RT by a sigmoidal relationship due to a blunted response of ischemia for short RTs. This result suggests that a minimal time of recovery is needed at the symptomatic lower extremity (muscle) level for the initiation of recovery from ischemia.

406 Nonetheless, the fact that the walking capacity started to recover at $40-50 \%$, even after 0.5 $\min$ of recovery while the end-recovery ischemia response was blunted for up to $2.5 \mathrm{~min}$ during recovery, suggests that the restoration of walking capacity seems independent of underlying ischemia, at least during the first 2 to $3 \mathrm{~min}$ of recovery. This result is consistent

410 with that in a previous study that showed that although the peak forces of forearm muscles 411 during an isometric hand-grip exercise declined more when blood flow was mechanically 
412 occluded than during a normally perfused condition, the peak force restoration rate during

413 recovery was rapid, starting as early as $0.5 \mathrm{~min}$ of recovery and ending by $5 \mathrm{~min}$ of recovery

414 (43). Although phosphocreatine recovery from exercise is impaired in people with PAD

415 compared to healthy subjects (28), the initial recovery of walking capacity under ischemia

416 could be at least partially explained by a rapid (although incomplete) resynthesis of high-

417 energy phosphate stores of the anaerobic fibers that support the energy demands of the

418 ischemic musculature while walking is resumed $(21,43)$. However, it should be considered

419 that the pattern of $\mathrm{TcPO}_{2}$ changes during recovery is delayed with respect to that of other

420 hemodynamic variables related to ischemia, i.e. systolic ankle pressure or ankle-to-brachial

421 index $(18,21)$. Consequently, it cannot be excluded that ischemia at the muscle level started

422 to resolve sooner than the $\mathrm{TcPO}_{2}$ at the skin level. Of note, the blunting response of ischemia

423 during the first few minutes of recovery has been reported in other studies in which $\mathrm{TcPO}_{2}$

424 measurements were made during a single maximal walking bout followed by a single

425 recovery period $(5,18-20,35)$.

426

427 Our experimental conditions based on the isolated (TW-ISO) or consecutive (TW-CONS and

428 OW-CONS) repetitions of walking bout(s) inducing ischemia, followed by recovery bouts of

429 different durations, could be reflected by a very short-term and acute model of repeated

430 ischemia-reperfusion cycles (42). The ischemia-reperfusion (I/R) process causes local and

431 distant changes ( $\mathrm{I} / \mathrm{R}$ injury) due to a number of pathophysiological events that occur during

432 the ischemic phase (i.e., the production of reactive oxygen species, inflammation and

433 mitochondrial dysfunction) and that amplify damage in the previously ischemic tissues when

434 blood flow recirculates during the reperfusion phase (42). In the long term, acute repetitions

435 of this ischemia-reperfusion cycle impair the exercising skeletal muscle function, which

436 further limits the walking capacity of people with PAD (25). The chronology of deleterious 
437 events involved in I/R injuries have been mainly investigated using experimental models that

438 included several hours of ischemia (42). An acute bout of treadmill walking has been shown

439 to trigger an excessive inflammatory response and oxidative stress leading to endothelium

440 damage, suggesting that short-term I/R injuries are exercise induced in people with PAD

441 (11). However, it is unknown whether such deleterious events delay walking capacity

442 restoration until a given recovery time is reached, as shown here; this topic should be studied

443 further. Finally, remote ischemic preconditioning has been shown to protect against $\mathrm{I} / \mathrm{R}$

444 injuries (34), but the results regarding its acute effect on walking capacity in people with

445 PAD are inconsistent $(10,47)$. Our results showed there was no ischemic preconditioning

446 effect during acute repeated walking bouts since the restoration of MWT was capped at

447 approximately $100 \%$, even with longer RTs.

449 Effect of RT on walking capacity restoration: inter-subjects and inter-conditions variability

450 Although most of the participants with PAD exhibited a logarithmic effect of RT on walking 451 capacity, few patients had a linear profile ( $n=1$ to 3 depending on the walking condition). 452 This effect may have been logarithmic if longer RTs had been tested in those participants 453 with a linear profile. However, this approach would have increased the session duration and 454 the number of walking bouts to be performed, particularly for the TW-CONS and OW-CONS 455 conditions, and the accumulated fatigue could have exerted a major confounding effect on 456 walking capacity (31). The different profiles reported here seem consistent with the inter457 individual variability in the time course of recovery from ischemia, as previously reported 458 (48). The linear profile implies that some people with PAD need $>10$ min to recover from 459 ischemia after a maximal walking bout, independently of lower-limb symptom dissipation, 460 supporting the mismatch between time to symptom relief and resolution of ischemia $(14,48)$. 
461 An important result was that the effect of RT on walking capacity was observed in three

462 different experimental conditions. However, there were some different results between the 463 tested experimental conditions. First, the RT needed to reach full restoration of walking 464 capacity and full recovery of ischemia was longer for TW-CONS than for TW-ISO. This can 465 be explained by the consecutive nature of the walking bouts performed: the time needed to 466 recover from a given walking bout limited by ischemia could be extended due to the 467 accumulation of previous walking bout(s), inducing slower adaptation of muscle blood flow 468 (12) and/or slower phosphocreatine recovery (28). Interestingly, assuming that cumulated 469 time is an acceptable tool for estimating fatigue, there was no negative association between 470 the cumulated time and walking capacity for all but one participant. This result suggests that 471 RT was the more predominant and powerful predictor of walking capacity during TW472 CONS. From a practical point of view, this is an important finding in view of defining a 473 feasible procedure to determine the effect of RT on walking capacity, as well as possibly 474 determining the IRT at the individual level. Second, compared with during TW-CONS, 475 during OW-CONS, participants with PAD were unable to restore their MWT to the same 476 extent. This was an unexpected observation. Indeed, because the intensity of outdoor walking 477 for people with PAD is lower outdoors than it is on the treadmill $(29,31)$, we could expect a 478 lower level of ischemia at the end of each outdoor walking bout (14), thereby allowing faster 479 restoration of walking capacity. Participants with PAD actually walked longer during OW480 CONS due to a lower walking intensity compared with that under TW conditions, which 481 required some participants to stop the session before testing all RTs to avoid an excessive 482 walking session duration. The low level of restoration was likely to be explained by the 483 longer walking duration that led to a marked level of ischemia and that would also have 484 required more than 5 min of recovery. We previously reported that the cumulative time could 
485 also account for walking capacity changes during an outdoor walking session (31). This was

486 not the case in the present study, as this was not the case for walking speed.

Study limitations

490 For a few participants (3 for TW-ISO, 4 for TW-CONS, 7 for OW-CONS), no significant 491 effect of RT on walking capacity was found $(P>0.05)$. Visually, the effect was observable, 492 but the issue was statistical due to the presence of outlier(s), and a limited number of points 493 (RTs) were available to build individual models. Furthermore, the absence of an effect would 494 have meant an absence of the underlying physiological phenomenon for these participants, 495 which should have been consistent across the three experimental conditions; this was not the 496 case.

497 Furthermore, the absence of a physiological measurement of ischemia during OW-CONS 498 made it difficult to ascertain whether the changes in walking capacity were due to ischemia or 499 to other fatigue-related mechanisms (31). Portable $\mathrm{TcPO}_{2}$ devices for ambulatory measures in 500 the field are not available, but near-infrared spectroscopy may be used for such 501 measurements (40).

Future directions

504 "Should I stay or should I go now?" That is a core question that people with PAD may ask 505 themselves when they stop walking due to ischemic lower-limb pain and rest for a while 506 before resuming walking. The same question can be asked by clinicians when controlling the 507 RT during a rehabilitation session since the available recommendations are limited $(4,6,17$, 508 22, 52). The present study is only the first step, and complementary studies are needed before 509 a full response to this question can be provided. However, the present study provides 
510 benchmarks for clinicians or physical therapists on how to manage RT, which can be 511 summarized as follows:

512 i) A median work-to-rest ratio $\geq 1: 1$ implied the resumption of walking with a 513 substantial level of ischemia and did not allow the restoration of MWT by more $514 \quad$ than $80 \%$ at the group level.

515 ii) A median work-to-rest ratio $\leq 1: 2$ seemed to be necessary to allow full recovery 516 from ischemia and the restoration of $\sim 100 \%$ MWT at the group level.

517 iii) The IRT, which is a new concept in terms of RT that we introduced here, was 518 associated with a median work-to-rest ratio between 1:1 and 1:2, which allowed 519 the symptomatic lower-limb muscle to start to recover from ischemia and the

iv) The median SSRT, as well as the median time needed to reach a pain level of " 0 " (which was similar to the SSRT), corresponded to median work-to-rest ratios of $>1: 1$, meaning that participants with PAD spontaneously resumed walking following a RT that was clearly insufficient in terms of recovery from ischemia, which precluded the restoration of MWT above $80 \%$.

526 During rehabilitation programs, pain dissipation is currently used as the main criterion to 527 define the between-walking-bouts recovery duration $(6,26,52)$. Our results confirm the well528 known dissociation between ischemia dissipation and symptom relief during recovery (13, 529 14), but the results show for the first time the detrimental effect of spontaneously resuming 530 walking on subsequent walking capacity after a walking stop induced by ischemic pain. Thus, 531 if the clinician aims to maximize walking restoration with full ischemia recovery after each 532 walking bout, it appears that the use of the SSRT or time to pain " 0 " is likely to be 533 inappropriate. However, clinicians often aim to maximize the time spent walking during each 534 session, and the ideal RT for such a purpose remains a core issue. Additional future studies 
535 are required to compare the use of the SSRT, IRT, or time to reach full walking capacity

536 recovery for maximizing the time spent walking within different session durations. An

537 important point also to consider is that our results regarding the relationship between RT and

538 walking capacity were obtained from walking bouts performed up to maximal claudication

539 pain, whereas in the majority of supervised exercise treadmill programs, patients are

540 instructed to walk to moderate or near-maximal level of claudication $(24,52)$. Although they

541 are experimentally challenging to conduct, studies on the relationship between different

542 work-to-rest ratios and walking capacity restoration according to various pain endpoints

543 should be conducted to identify optimal and maybe new walking training regimens, while

544 minimizing the acute adverse responses of exercise up to maximal claudication pain (e.g.,

545 inflammation, denervation), even if these adverse effects are overtaken by the benefits of

546 chronic exercise (24). Finally, besides the current recommendations on what is considered the

547 "optimal" exercise regimen $(6,52)$, our results may not be applicable in other context of

548 walking programs and that have proven to be efficient, e.g. programs with fixed work:rest

549 ratios and fixed walking speeds (36), those based on interval training with active recovery

550 (53) or those based on pain-free treadmill training (38).

551 In addition to rehabilitation programs, different strategies of recovery that vary by daily life

552 contexts could be of interest for people with PAD and could be studied in the future. Based

553 on our results, future studies should address whether the SSRT, the IRT or the time for full

554 recovery helps people with PAD manage pain according to specific contexts. Regardless,

555 managing RT from walking-induced ischemia during daily life could be facilitated through

556 the use of wearable monitors (7).

557 Future studies are also needed to determine the reliability of IRT. Finally, we are aware of no 558 study on the impact of walking duration on ischemia independently of the effect of walking 
559 intensity and are likewise unaware of any previous implementation of ambulatory ischemia

560 measurements during outdoor walking.

561

\section{CONCLUSION}

563 This study highlighted the significant and mostly logarithmic effect of RT on walking 564 capacity in people with symptomatic PAD. Moreover, this study revealed that (i) self565 selection of RT or the time to reach pain " 0 " corresponding to a median work-to-rest ratio

566 higher than 1:1 leads to the resumption of walking with unrecovered ischemia and precludes 567 restoration of full walking capacity; and (ii) it is possible to determine an individualized RT

568 allowing the initiation of recovery from ischemia and better restoration of the walking 569 capacity, while a work-to-rest ratio of $\leq 1: 2$ should be preferred to fully restore the walking 570 capacity.

571 
REFERENCES

573 1. Aboyans V, Criqui MH, Abraham P, Allison MA, Creager MA, Diehm C,

574 Fowkes FG, Hiatt WR, Jonsson B, Lacroix P, Marin B, McDermott MM, Norgren L,

575 Pande RL, Preux PM, Stoffers HE, Treat-Jacobson D, on behalf of the American Heart

576 Association Council on Peripheral Vascular Disease, Council on Epidemiology

577 Prevention, Council on Clinical Cardiology, Council on Cardiovascular Nursing,

578 Council on Cardiovascular Radiology Intervention, and Council on Cardiovascular

579 Surgery Anesthesia. Measurement and interpretation of the ankle-brachial index: a scientific

580 statement from the American Heart Association. Circulation 126: 2890-2909, 2012.

581 2. Abraham P, Picquet J, Vielle B, Sigaudo-Roussel D, Paisant-Thouveny F, Enon

582 B, and Saumet JL. Transcutaneous oxygen pressure measurements on the buttocks during

583 exercise to detect proximal arterial ischemia: comparison with arteriography. Circulation

584 107: 1896-1900, 2003.

585 3. American College of Sports Medicine. ACSM's Guidelines for Exercise Testing and

586 Prescription. Philadelphia, PA: Wolters Kluwer/Lippincott Williams \& Wilkins, 2014.

587 4. Askew CD, Parmenter B, Leicht AS, Walker PJ, and Golledge J. Exercise \&

588 Sports Science Australia (ESSA) position statement on exercise prescription for patients with

589 peripheral arterial disease and intermittent claudication. J Sci Med Sport 17: 623-629, 2014.

$590 \quad$ 5. Bouye P, Picquet J, Jaquinandi V, Enon B, Leftheriotis G, Saumet JL, and

591 Abraham P. Reproducibility of proximal and distal transcutaneous oxygen pressure

592 measurements during exercise in stage 2 arterial claudication. Int Angiol 23: 114-121, 2004.

593 6. Bulmer AC, and Coombes JS. Optimising exercise training in peripheral arterial

594 disease. Sports Med 34: 983-1003, 2004.

595 7. Chaudru S, Jehannin P, de Müllenheim PY, Klein H, Jaquinandi V, Mahé G,

596 and Le Faucheur A. Using wearable monitors to assess daily walking limitations induced by 
ischemic pain in peripheral artery disease. Scandinavian journal of medicine \& science in sports 29: 1813-1826, 2019.

8. Creager MA, Belkin M, Bluth EI, Casey DE, Jr., Chaturvedi S, Dake MD, Fleg

Jacobson D, Upchurch GR, Jr., White CJ, and Ziffer JA. 2012

ACCF/AHA/ACR/SCAI/SIR/STS/SVM/SVN/SVS Key data elements and definitions for peripheral atherosclerotic vascular disease: a report of the American College of Cardiology

Foundation/American Heart Association Task Force on clinical data standards (Writing

608 9. Criqui MH, Denenberg JO, Bird CE, Fronek A, Klauber MR, and Langer RD.

609 The correlation between symptoms and non-invasive test results in patients referred for

610 peripheral arterial disease testing. Vasc Med 1: 65-71, 1996.

611 10. Delagarde H, Ouadraougo N, Grall S, Macchi L, Roy PM, Abraham P, and

612 Prunier F. Remote ischaemic preconditioning in intermittent claudication. Arch Cardiovasc

613 Dis 108: 472-479, 2015.

614 11. Edwards AT, Blann AD, Suarez-Mendez VJ, Lardi AM, and McCollum CN.

615 Systemic responses in patients with intermittent claudication after treadmill exercise. $\mathrm{Br} J$

616 Surg 81: 1738-1741, 1994.

617 12. Faisal A, Dyson KS, and Hughson RL. Prolonged ischaemia impairs muscle blood

618 flow and oxygen uptake dynamics during subsequent heavy exercise. J Physiol 588: 3785-

$6193797,2010$.

620 13. Fouasson-Chailloux A, Abraham P, Colas-Ribas C, Feuilloy M, Vielle B, and

621 Henni S. Simultaneous pain intensity rating and quantification of ischemia throughout 
622 exercise and recovery in proximal versus distal arterial claudication. Vasc Med 22: 490-497, 6232017.

624 14. Gardner AW. Dissipation of claudication pain after walking: implications for 625 endurance training. Med Sci Sports Exerc 25: 904-910, 1993.

626 15. Gardner AW, Montgomery PS, and Afaq A. Exercise performance in patients with 627 peripheral arterial disease who have different types of exertional leg pain. $J$ Vasc Surg 46: $62879-86,2007$.

629 16. Gardner AW, Parker DE, Montgomery PS, Scott KJ, and Blevins SM. Efficacy

630 of quantified home-based exercise and supervised exercise in patients with intermittent

631 claudication: a randomized controlled trial. Circulation 123: 491-498, 2011.

632 17. Gardner AW, and Poehlman ET. Exercise rehabilitation programs for the treatment 633 of claudication pain. A meta-analysis. JAMA 274: 975-980, 1995.

634 18. Gardner AW, Skinner JS, Cantwell BW, and Smith LK. Progressive vs single635 stage treadmill tests for evaluation of claudication. Med Sci Sports Exerc 23: 402-408, 1991.

636 19. Gardner AW, Skinner JS, and Smith LK. Effects of handrail support on

637 claudication and hemodynamic responses to single-stage and progressive treadmill protocols

638 in peripheral vascular occlusive disease. Am J Cardiol 68: 99-105, 1991.

639 20. Gardner AW, Skinner JS, Vaughan NR, Bryant CX, and Smith LK. Comparison

640 of three progressive exercise protocols in peripheral vascular occlusive disease. Angiology

641 43: 661-671, 1992.

642 21. Gardner AW, Skinner JS, Vaughan NR, Bryant CX, and Smith LK. Comparison

643 of treadmill walking and stair climbing over a range of exercise intensities in peripheral

644 vascular occlusive disease. Angiology 44: 353-360, 1993.

645 22. Gerhard-Herman MD, Gornik HL, Barrett C, Barshes NR, Corriere MA, 646 Drachman DE, Fleisher LA, Fowkes FGR, Hamburg NM, Kinlay S, Lookstein R, Misra 
Stewart KJ, Treat-Jacobson D, and Walsh ME. 2016 AHA/ACC Guideline on the

management of patients with lower extremity peripheral artery disease: a report of the

American College of Cardiology/American Heart Association Task Force on clinical practice guidelines. Journal of the American College of Cardiology 69: e71-e126, 2017.

\section{Gernigon M, Le Faucheur A, Noury-Desvaux B, Mahe G, Abraham P, and Post-}

GPS Study Coinvestigators Group. Applicability of global positioning system for the assessment of walking ability in patients with arterial claudication. J Vasc Surg 60: 973-981 e971, 2014.

24. Hamburg NM, and Balady GJ. Exercise rehabilitation in peripheral artery disease: functional impact and mechanisms of benefits. Circulation 123: 87-97, 2011.

25. Hiatt WR, Armstrong EJ, Larson CJ, and Brass EP. Pathogenesis of the limb manifestations and exercise limitations in peripheral artery disease. Circ Res 116: 1527-1539, 6602015.

26. Hirsch AT, Haskal ZJ, Hertzer NR, Bakal CW, Creager MA, Halperin JL, Hiratzka LF, Murphy WR, Olin JW, Puschett JB, Rosenfield KA, Sacks D, Stanley JC,

Ornato JP, Page RL, and Riegel B. ACC/AHA 2005 Practice guidelines for the management of patients with peripheral arterial disease (lower extremity, renal, mesenteric, and abdominal aortic): a collaborative report from the American Association for Vascular Surgery/Society for Vascular Surgery, Society for Cardiovascular Angiography and Interventions, Society for Vascular Medicine and Biology, Society of Interventional Radiology, and the ACC/AHA Task Force on practice guidelines (writing committee to

671 develop guidelines for the management of patients with peripheral arterial disease): endorsed 
672 by the American Association of Cardiovascular and Pulmonary Rehabilitation; National

673 Heart, Lung, and Blood Institute; Society for Vascular Nursing; TransAtlantic Inter-Society

674 Consensus; and Vascular Disease Foundation. Circulation 113: e463-654, 2006.

675 27. Hogan MC, Richardson RS, and Kurdak SS. Initial fall in skeletal muscle force

676 development during ischemia is related to oxygen availability. J Appl Physiol (1985) 77:

$677 \quad 2380-2384,1994$.

678 28. Kemp GJ, Roberts N, Bimson WE, Bakran A, Harris PL, Gilling-Smith GL,

679 Brennan J, Rankin A, and Frostick SP. Mitochondrial function and oxygen supply in

680 normal and in chronically ischemic muscle: a combined 31P magnetic resonance

681 spectroscopy and near infrared spectroscopy study in vivo. J Vasc Surg 34: 1103-1110, 2001.

682 29. Le Faucheur A, Abraham P, Jaquinandi V, Bouye P, Saumet JL, and Noury-

683 Desvaux B. Measurement of walking distance and speed in patients with peripheral arterial

684 disease: a novel method using a global positioning system. Circulation 117: 897-904, 2008.

685 30. Le Faucheur A, Abraham P, Jaquinandi V, Bouye P, Saumet JL, and Noury-

686 Desvaux B. Study of human outdoor walking with a low-cost GPS and simple spreadsheet

687 analysis. Med Sci Sports Exerc 39: 1570-1578, 2007.

688 31. Le Faucheur A, Noury-Desvaux B, Mahe G, Sauvaget T, Saumet JL, Leftheriotis

689 G, and Abraham P. Variability and short-term determinants of walking capacity in patients

690 with intermittent claudication. J Vasc Surg 51: 886-892, 2010.

691 32. Leeper NJ, Myers J, Zhou M, Nead KT, Syed A, Kojima Y, Caceres RD, and

692 Cooke JP. Exercise capacity is the strongest predictor of mortality in patients with peripheral

693 arterial disease. J Vasc Surg 57: 728-733, 2013.

694 33. Leplege A, Ecosse E, Verdier A, and Perneger TV. The French SF-36 Health

695 Survey: translation, cultural adaptation and preliminary psychometric evaluation. J Clin

696 Epidemiol 51: 1013-1023, 1998. 
and MacAllister RJ. Remote ischemic preconditioning provides early and late protection system. J Am Coll Cardiol 46: 450-456, 2005.

35. Mahe G, Kalra M, Abraham P, Liedl DA, and Wennberg PW. Application of exercise transcutaneous oxygen pressure measurements for detection of proximal lower extremity arterial disease: a case report. Vasc Med 20: 251-255, 2015.

36. Manfredini F, Lamberti N, Guerzoni F, Napoli N, Gasbarro V, Zamboni P,

Mascoli F, Manfredini R, Basaglia N, Rodriguez-Borrego MA, and Lopez-Soto PJ.

Rehabilitative exercise reduced the impact of peripheral artery disease on vascular outcomes in elderly patients with claudication: a three-year single center retrospective study. J Clin Med 8: 2019.

709 37. McDermott MM, Liu K, Guralnik JM, Martin GJ, Criqui MH, and Greenland

710 P. Measurement of walking endurance and walking velocity with questionnaire: validation of

711 the walking impairment questionnaire in men and women with peripheral arterial disease. $J$

712 Vasc Surg 28: 1072-1081, 1998.

713 38. Mika P, Konik A, Januszek R, Petriczek T, Mika A, Nowobilski R, Nizankowski

714 R, and Szczeklik A. Comparison of two treadmill training programs on walking ability and 715 endothelial function in intermittent claudication. Int J Cardiol 168: 838-842, 2013.

716 39. Muggeo VMR. Segmented: an R package to fit regression models with broken-line 717 relationships. $R$ News 8: 20-25, 2008.

718 40. Murrow JR, Brizendine JT, Djire B, Young H-J, Rathbun S, Nilsson KR, Jr., 719 and McCully KK. Near infrared spectroscopy-guided exercise training for claudication in 720 peripheral arterial disease. European journal of preventive cardiology 26: 471-480, 2019. 
721 41. Noury-Desvaux B, Abraham P, Mahe G, Sauvaget T, Leftheriotis G, and Le

722 Faucheur A. The accuracy of a simple, low-cost GPS data logger/receiver to study outdoor

723 human walking in view of health and clinical studies. PLoS One 6: e23027, 2011.

724 42. Paradis S, Charles AL, Meyer A, Lejay A, Scholey JW, Chakfe N, Zoll J, and

725 Geny B. Chronology of mitochondrial and cellular events during skeletal muscle ischemia-

726 reperfusion. Am J Physiol Cell Physiol 310: C968-982, 2016.

727 43. Pitcher JB, and Miles TS. Influence of muscle blood flow on fatigue during

728 intermittent human hand-grip exercise and recovery. Clin Exp Pharmacol Physiol 24: 471-

$729476,1997$.

730 44. Poulin A, Guilcher A, Omarjee L, Jaquinandi V, Gandon Y, Mahe G, and

731 Stivalet $\mathbf{O}$. Validation of a software to perform exercise oximetry to diagnose arterial

732 stenosis of the lower limbs. Atherosclerosis 278: 325-327, 2018.

733 45. R Core Team. R: A language and environment for statistical computing. R

734 Foundation for Statistical Computing, Vienna, Austria. URL https:/www.R-project.org/.

7352019.

736 46. Regensteiner JG, Hiatt WR, Coll JR, Criqui MH, Treat-Jacobson D, McDermott

737 MM, and Hirsch AT. The impact of peripheral arterial disease on health-related quality of

738 life in the Peripheral Arterial Disease Awareness, Risk, and Treatment: New Resources for

739 Survival (PARTNERS) program. Vasc Med 13: 15-24, 2008.

740 47. Saes GF, Zerati AE, Wolosker N, Ragazzo L, Rosoky RM, Ritti-Dias RM,

741 Cucato GG, Chehuen M, Farah BQ, and Puech-Leão P. Remote ischemic preconditioning

742 in patients with intermittent claudication. Clinics (Sao Paulo) 68: 495-499, 2013.

743 48. Schorr EN, Treat-Jacobson D, and Lindquist R. The relationship between

744 peripheral artery disease symptomatology and ischemia. Nurs Res 66: 378-387, 2017. 
746 Global, regional, and national prevalence and risk factors for peripheral artery disease in

747 2015: an updated systematic review and analysis. Lancet Glob Health 7: e1020-e1030, 2019.

748 50. Sorlie D, and Myhre K. Lower leg blood flow in intermittent claudication. Scand J

749 Clin Lab Invest 38: 171-179, 1978.

750 51. Stivalet O, Paisant A, Belabbas D, Omarjee L, Le Faucheur A, Landreau P,

751 Garlantezec R, Jaquinandi V, Liedl DA, Wennberg PW, and Mahe G. Exercise testing

752 criteria to diagnose lower extremity peripheral artery disease assessed by computed-

753 tomography angiography. PLoS One 14: e0219082, 2019.

754 52. Treat-Jacobson D, McDermott MM, Bronas UG, Campia U, Collins TC, Criqui

755 MH, Gardner AW, Hiatt WR, Regensteiner JG, Rich K, on behalf of the American

756 Heart Association Council on Peripheral Vascular Disease, Council on Quality of Care

757 and Outcomes Research, and Council on Cardiovascular and Stroke Nursing. Optimal

758 exercise programs for patients with peripheral artery disease: a scientific statement from the

759 American Heart Association. Circulation 139: e10-e33, 2019.

760 53. Villemur B, Thoreau V, Guinot M, Gailledrat E, Evra V, Vermorel C, Foote A,

761 Carpentier P, Bosson JL, and Perennou D. Short interval or continuous training programs

762 to improve walking distance for intermittent claudication: pilot study. Ann Phys Rehabil Med

763 63: 466-473, 2020.

764 
Figure 1. Schematic view of the experimental conditions tested in the protocol

Note. TW-ISO = Isolated sequences of treadmill maximal walking bouts; TW-CONS =

769 Consecutive treadmill maximal walking bouts; OW-CONS = Consecutive outdoor maximal

770 walking bouts; $\mathrm{W}=$ Walk; $\mathrm{R}=$ Recovery; SSRT $=$ Self-selected recovery time; $\mathrm{TcPO}_{2}=$

771 Transcutaneous oxygen pressure; GPS = Global Positioning System.

772

773

Figure 2. Illustration of the procedure to determine the individual recovery time (IRT)

in one patient following the TW-ISO condition. The Self-selected recovery time (SSRT)

is also displayed.

776

777

Figure 3. Effect of the recovery time on the restoration of walking capacity and endrecovery physiological variables

Note. The figure displays the data related only to participants who exhibited a plausible

780 logarithmic or linear effect of recovery time (RT) on walking capacity $(P \leq 0.05$ for the slope).

781 TW-ISO $=$ Isolated sequences of treadmill maximal walking bouts; $\mathrm{TW}$-CONS =

782 Consecutive treadmill maximal walking bouts; OW-CONS = Consecutive outdoor maximal

783 walking bouts; SSRT $=$ Self-selected RT; IRT $=$ Individual RT; Walk $\mathrm{N}_{\mathrm{N}}=$ Walking time up to

784 maximal claudication pain performed after the RT tested; Walk F $_{1}$ First walking time up to

785 maximal claudication pain during the walking sequence; $\% \dot{\mathrm{V}} \mathrm{O}_{2 \text { peak }}=$ Percentage of peak

786 oxygen uptake; $\% \mathrm{HR}_{\text {peak }}=$ Percentage of peak heart rate; DROP $=$ Delta from resting

787 transcutaneous oxygen pressure; $\mathrm{PFWT}=$ Pain-free walking time; $\mathrm{MWT}=$ Maximal walking 788 time. 
789 The left, middle, and right panels are related to the TW-ISO, TW-CONS, and OW-CONS

790 conditions, respectively. For panels $\mathrm{G}$ to $\mathrm{L}$, dashed lines and gray bands represent the

791 medians of the participants' resting values and their respective interquartile ranges for the

792 physiological parameters considered. Resting $\dot{\mathrm{VO}}_{2}$ and $\mathrm{HR}$ values were determined as the

793 lowest mean values of $\dot{\mathrm{V}}{ }_{2}$ and $\mathrm{HR}$ obtained among all the 2-min resting periods (treadmill

794 and outdoor sessions included) of the protocol, respectively.

795 For TW-ISO, one datum was missing for PFWT and DROP, respectively, for the RTs of 6.5

796 and $3.5 \mathrm{~min}$. For TW-CONS, one datum was missing for PFWT for the RT of $0.5 \mathrm{~min}$. For

797 OW-CONS, one datum was missing for PWFT for each of the tested RTs. Data were missing

798 due to either no communication of pain onset by the participant (for PFWT) or device

799 malfunction (for DROP).

$800 * P \leq 0.05, * * P \leq 0.01, * * * P \leq 0.001$, compared with the previous RT. 


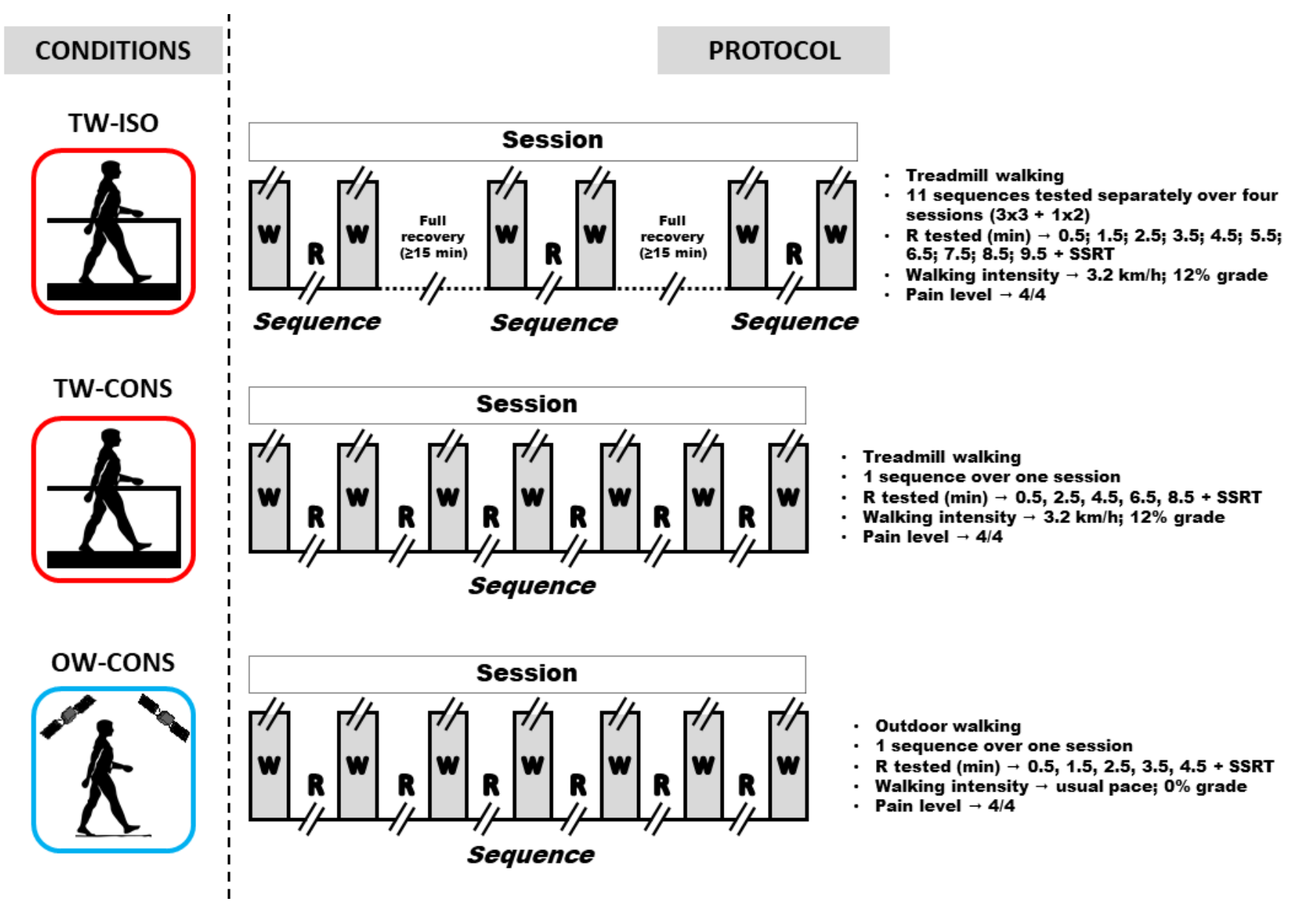

\section{MEASURES}

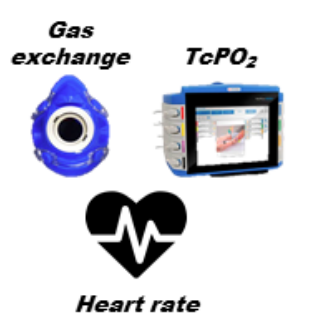

Gas

exchange $\mathrm{TcPO}_{2}$

(O)
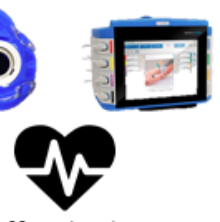

Heart rate

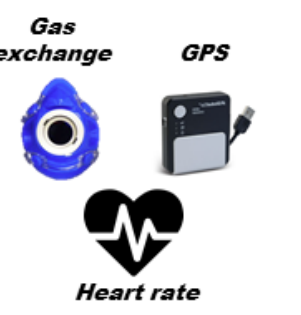




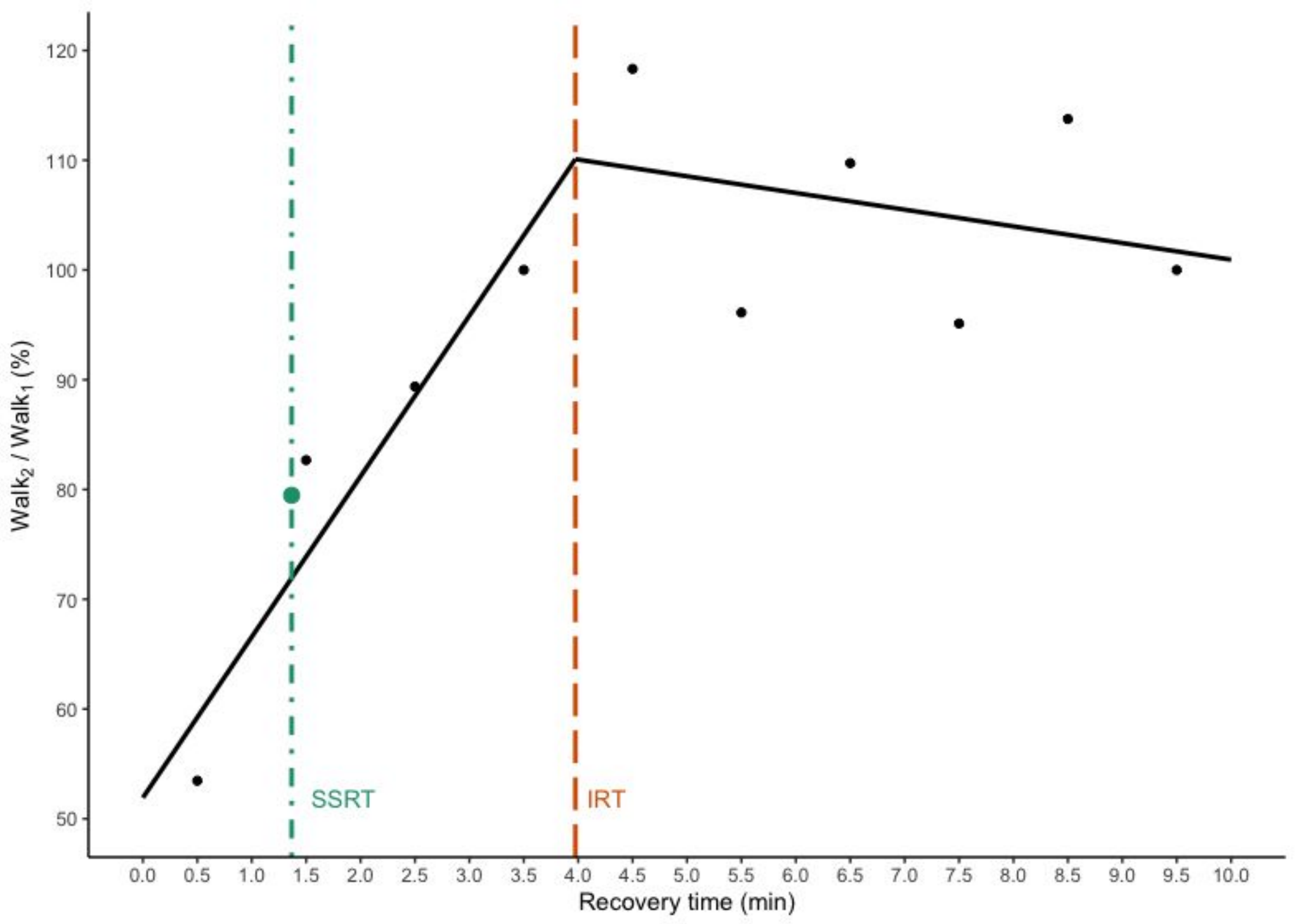


Table 1. Inclusion and non-inclusion criteria of the CLASH study

\section{Inclusion criteria}

- $\quad$ Age $\geq 18$ years old

- Insured under the French social security system (according to French law)

- Presence of lower-extremity peripheral artery disease, defined by:

- A resting ankle-brachial index $(\mathrm{ABI}) \leq 0.90$

$\circ$ OR if resting $\mathrm{ABI}>0.90$ and $<1.00$, a decrease in recovery ankle systolic pressure or in recovery $\mathrm{ABI}$ from treadmill exercise greater than $30 \%$ or $20 \%$, respectively (AHA recommendations(1)).

○ OR if resting $\mathrm{ABI}>1.40$, a toe pressure index $\leq 0.70$

- Maximal walking distance on treadmill $(3.2 \mathrm{~km} / \mathrm{h}, 10 \%$ grade $)<500 \mathrm{~m}^{\dagger}$

- Complaint of exertional lower limb pain that can sometimes begin at rest, causing the participant to stop walking, and is relieved or lessened within 10 minutes of rest (confirmed during treadmill testing)

\section{Non-inclusion criteria}

- Exercise limitation due to symptoms not related to an arterial insufficiency in the lower limbs (e.g., dyspnea, angina pectoris)

- Contraindication for walking (abdominal aortic aneurysm $>4 \mathrm{~cm}$ )

- Myocardial infarction and/or stroke in the last 3 months

- Critical limb ischemia, amputation

- Pregnant women

- Adult subject to legal protection (guardianship or tutelage measure) and persons deprived from their liberty (according to French law).

- People living more than $50 \mathrm{~km}$ from the university hospital

- People unable to understand the instructions of the study 
${ }^{\dagger}$ As assessed during the medical appointment.

${ }^{\ddagger}$ According to our inclusion criteria, PAD participants with leg symptoms that fell within the following leg symptom categories could be included in the study:

i) Intermittent claudication. People with PAD who experience exertional leg pain that does not begin at rest and that forces them to stop walking and that relieves or lessens within 10 minutes of rest;

ii) Leg pain on exertion and rest. In this category, people with PAD experience exertional lower limb pain during walking, but this pain can sometimes begin at rest when they are standing still or sitting. As noted by Criqui et al. (10), this category of people with PAD with "pain at rest" should not be confused with those who experience "resting pain", which usually refers to people with such severe advanced PAD that ischemic pain is present even at rest. Subjects with ischemic rest pain were not included in the study. 
Table 2. Characteristics of PAD participants

\section{General}

Age, y

$60 \pm 10$

Height, $\mathrm{m}$

$1.69 \pm 0.08$

Weight, kg

$77 \pm 15$

Body mass index, $\mathrm{kg} \cdot \mathrm{m}^{-2}$

$27 \pm 4$

Waist circumference, $\mathrm{cm}$

$101 \pm 11$

Women, n (\%)

$3(14)$

Caucasian, $\mathrm{n}(\%)$

$21(100)$

Former smokers, n (\%)

$11(52)$

Current smokers, n (\%)

$10(48)$

\section{Comorbid conditions}

History of diabetes mellitus, n (\%)

$5(24)$

History of hypertension, n (\%)

$13(62)$

History of heart failure, n (\%)

$3(14)$

History of myocardial infarction/stable angina, n (\%)

$3(14)$

History of stroke, n (\%)

$1(5)$

History of cancer, $\mathrm{n}(\%)$

$1(5)$

History of chronic obstructive pulmonary disease, n (\%)

$6(29)$

\section{PAD condition}

Ankle-brachial index

$0.67 \pm 0.17$

Leg symptoms ${ }^{\dagger}, \mathrm{n}(\%)$

Intermittent claudication

Leg pain on exertion and rest

Atypical claudication

$1(5)$ 
Previous lower-extremity revascularization

$7(33)$

\section{Medications}

Anti-diabetics, n (\%)

$5(24)$

Antihypertensive drugs, n (\%)

$12(57)$

Antiplatelet agents or Anticoagulants, n (\%)

$19(90)$

Cholesterol-lowering drug, n (\%)

$19(90)$

$\beta$-blockers, n (\%)

Vasodilators, n (\%)

$3(14)$

\section{Walking capacity}

WIQ scores

WIQ distance score, $\%$

$23(14-40)$

WIQ speed score, $\%$

$25(17-33)$

WIQ stairs score, $\%$

$63(25-75)$

WIQ total score, $\%$

$37(21-49)$

Maximal walking distance

$\mathrm{MWD}_{\mathrm{SR}}, \mathrm{m}^{\S}$

$133(100-250)$

MWD GARDNER, $\mathrm{m}$

$265(171-364)$

MWD6WT, $\mathrm{m}$

$384(339-433)$

MWDGPS, $^{m}$

$430(298-529)$

\section{Peak physiological responses}

Peak $\dot{\mathrm{VO}} 2, \mathrm{~mL} \cdot \mathrm{min}^{-1} \cdot \mathrm{kg}^{-1}$

$16.03 \pm 2.22$

Peak HR, bpm

$108 \pm 18$ 
For the purpose of the CLASH study, a six-minute walking test was also performed according to previous recommendations (4). This test was performed at the beginning of the TW-ISO session and included only two sequences. Sufficient recovery time ( $>30 \mathrm{~min})$ was allowed following the six-minute walking test. Although normally distributed, the 6min total walking distance is shown using the median and interquartile range to allow easier comparisons with the other walking capacity outcomes. $\mathrm{PAD}=$ peripheral artery disease; WIQ = walking impairment questionnaire; $\mathrm{MWDSR}_{\mathrm{SR}}=$ self-reported maximal walking distance; MWDGARDNER $_{\text {G }}$ maximal walking distance measured during the Gardner-Skinner treadmill test; $\mathrm{MWD}_{6 \mathrm{WT}}=$ total walking distance measured during the 6min walking test; $\mathrm{MWD}_{\mathrm{GPS}}=$ highest maximal walking distance measured using the Global Positioning System during the outdoor walking session; $\mathrm{HR}=$ heart rate; $\dot{\mathrm{V}} \mathrm{O}_{2}=$ oxygen uptake.

${ }^{\dagger}$ Leg symptoms reported using the San Diego claudication questionnaire; see Table 1 for definition. ${ }^{*}$ IQ scores range between 0 and $100 \%$, with $100 \%$ indicating no impairment. ${ }^{\S}$ Data were available for 18 participants because 3 participants could not estimate their maximal walking distance. "Data correspond to the Gardner-Skinner treadmill test. 
Table 3. Summary of the group-based (mixed) models tested for the effect of recovery time on walking capacity $\left(\mathrm{MWT}_{\mathrm{n}} / \mathrm{MWT}_{1}\right.$, in \%)

\begin{tabular}{|c|c|c|c|c|c|c|}
\hline & \multicolumn{2}{|c|}{ TW-ISO $(n=21)$} & \multicolumn{2}{|c|}{ TW-CONS $(n=21)$} & \multicolumn{2}{|c|}{ OW-CONS $(n=20) *$} \\
\hline & $\mathbf{x}$ & $\ln (x)$ & $\mathbf{x}$ & $\ln (x)$ & $\mathbf{x}$ & $\ln (x)$ \\
\hline $\mathrm{R}^{2}$ & 0.19 & 0.23 & 0.34 & 0.45 & 0.22 & 0.24 \\
\hline $\mathrm{p}$-value & $2.59 \cdot 10^{-12}$ & $4.70 \cdot 10^{-15}$ & $5.47 \cdot 10^{-18}$ & $2.78 \cdot 10^{-29}$ & $2.15 \cdot 10^{-13}$ & $1.11 \cdot 10^{-15}$ \\
\hline RMSE & 24.51 & 24.08 & 21.27 & 19.50 & 35.56 & 35.09 \\
\hline \multicolumn{6}{|c|}{$\mathrm{x}$ and $\ln (\mathrm{x})$ are the explanatory variables that have been used in the mixed models, $\mathrm{x}$ being the } & recovery time. TW-ISO = isolated sequences of maximal treadmill walking bouts; TW-CONS \\
\hline \multicolumn{7}{|c|}{$=$ single sequence of consecutive maximal treadmill walking bouts; OW-CONS $=$ single } \\
\hline \multicolumn{7}{|c|}{ sequence of consecutive maximal outdoor walking bouts; $n=$ number of participants used for } \\
\hline \multicolumn{7}{|c|}{ the models; RMSE = root-mean-squared error (in \%: MWTn/MWT ${ }_{1}$ x 100). } \\
\hline \multicolumn{7}{|c|}{ *Data for only 20 participants were available for the models related to the CONS-OW } \\
\hline \multicolumn{7}{|c|}{ condition, as one participant did not stop in that condition. Moreover, in the OW-CONS } \\
\hline \multicolumn{7}{|c|}{ condition, two recovery times were not tested in three participants. These times were $1.5 \mathrm{~min}$} \\
\hline \multicolumn{7}{|c|}{ and $3.5 \mathrm{~min}$ for two participants and $2.5 \mathrm{~min}$ and the SSRT (not included in the analysis) for } \\
\hline
\end{tabular}


Table 4. Summary of the participant-based (individual) models for the effect of recovery time on walking capacity (MWT $/ \mathrm{MWT}_{1}$, in $\%$ )

\begin{tabular}{|c|c|c|c|c|c|c|}
\hline & \multicolumn{2}{|c|}{ TW-ISO } & \multicolumn{2}{|c|}{ TW-CONS } & \multicolumn{2}{|c|}{ OW-CONS } \\
\hline & $\mathbf{R}_{x}^{2}>\mathbf{R}_{\ln (x)}$ & $\mathbf{R}_{\ln (\mathrm{x})}^{2}>\mathbf{R}_{\mathrm{x}}^{2}$ & $\mathbf{R}_{x}^{2}>\mathbf{R}_{\ln (x)}^{2}$ & $\mathbf{R}_{\ln (\mathrm{x})}^{2}>\mathbf{R}_{\mathrm{x}}^{2}$ & $\mathbf{R}_{x}^{2}>\mathbf{R}_{\ln (x)}^{2}$ & $\mathbf{R}_{\ln (\mathrm{x})}^{2}>\mathbf{R}_{\mathrm{x}}^{2}$ \\
\hline $\mathrm{n}(\%)$ & $2(10)$ & $16(76)$ & $1(5)$ & $16(76)$ & $3(15)$ & $11(55)$ \\
\hline $\mathrm{R}^{2}$, median & 0.58 & 0.80 & 0.93 & 0.93 & 0.96 & 0.94 \\
\hline (IQR) & $(0.54-0.62)$ & $(0.70-0.87)$ & (n.a.) & $(0.88-0.97)$ & $(0.96-0.97)$ & $(0.88-0.96)$ \\
\hline SEE, median & 10.60 & 7.39 & 6.09 & 4.34 & 3.40 & 4.88 \\
\hline (IQR) & $(8.37-12.83)$ & $(6.61-10.79)$ & (n.a.) & $(3.60-5.93)$ & $(2.86-4.01)$ & $(4.27-6.11)$ \\
\hline
\end{tabular}

$\mathrm{R}_{\mathrm{x}}^{2}$ and $\mathrm{R}^{2} \ln (\mathrm{x})$ are the $\mathrm{R}^{2}$ coefficients related to models that respectively used either $\mathrm{x}$ or $\ln (\mathrm{x})$ as the explanatory variable, $\mathrm{x}$ being the recovery time. Statistics are shown only for the participants who exhibited a significant and plausible linear $\left(\mathrm{R}_{\mathrm{x}}^{2}>\mathrm{R}^{2}{ }_{\ln (\mathrm{x})}\right)$ or logarithmic $\left(\mathrm{R}^{2}{ }_{\ln (\mathrm{x})}>\mathrm{R}_{\mathrm{x}}^{2}\right)$ effect of recovery on walking capacity. TW-ISO = isolated sequences of maximal treadmill walking bouts; TW-CONS = single sequence of consecutive maximal treadmill walking bouts; OW-CONS = single sequence of consecutive maximal outdoor walking bouts; IQR = interquartile range; $\mathrm{SEE}=$ standard error of estimate; n.a. = not appropriate. 